\title{
Frequência e riqueza de cupins em áreas de plantio de eucalipto no litoral norte da Bahia
}

\author{
Maria José Dias Sales ${ }^{(1)}$, William Costa Matos $^{(2)}$, Yana Teixeira dos Reis ${ }^{(3)}$ e Genésio Tâmara Ribeiro(3)
}

\begin{abstract}
(1)Universidade Federal de Sergipe (UFS), Núcleo de Pós-Graduação e Estudos em Recursos Naturais, Campus Universitário, Avenida Marechal Rondon, s/no, Jardim Rosa Elze, CEP 49100-000 São Cristóvão, SE. E-mail: maria_uneb@yahoo.com.br (2)Copener Florestal Ltda., Rua Dr. José Tiago Correia, s/no, Alagoinhas Velha, CEP 48030-480 Alagoinhas, BA. E-mail: william_matos@bahiapulp.com (3)UFS, Centro de Ciências Biológicas e da Saúde. E-mail: yanatr@ufs.br, gribeiro@ufs.br
\end{abstract}

Resumo - O objetivo deste trabalho foi avaliar a frequência e a riqueza de espécies de cupins que ocorrem em áreas de reflorestamento com eucalipto. As amostras foram coletadas em três áreas de Eucalyptus recém-colhido, em dezembro de 2005, por meio de seis transectos de $100 \mathrm{~m}$ de comprimento por $2 \mathrm{~m}$ de largura, subdivididos em 20 parcelas $(2 \times 5 \mathrm{~m})$ contíguas. Cada parcela foi amostrada por uma hora por pessoa,

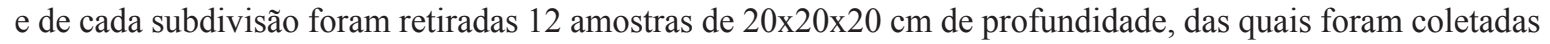
21 espécies de cupins, pertencentes a duas famílias e 16 gêneros. Dez espécies foram consideradas dominantes, todas da família Termitidae, das quais as de maior frequência foram Amitermes amifer e Nasutitermes corniger. O grupo funcional xilófago teve o maior número de espécies (11) e a maior frequência. Espécies conhecidas como pragas em eucalipto tiveram frequência abaixo do limite de dominância.

Termos para indexação: Amitermes amifer, Eucalyptus grandis, Nasutitermes corniger, térmitas, xilófagos.

\section{Frequency and species richness of termites in eucalyptus plantations in the north coast of Bahia, Brazil}

\begin{abstract}
The objective of this work was to evaluate the frequency and species richness of termites which occur in eucalyptus reforestation areas. The samples were collected from three recently harvested Eucalyptus areas, in December 2005, in $100 \mathrm{~m}$-long and $2 \mathrm{~m}$-wide transects, divided into 20 contiguous plots $(2 \times 5 \mathrm{~m})$. Each plot was sampled for one hour per person, and from each subdivision, 12 disturbed soil samples were taken from $20 \times 20 \times 20 \mathrm{~cm}$, from which 21 species of termites belonging to two families and 16 genera were collected. Ten species were found to be dominant, all belonging to the family Termitidae, from which those with the highest frequency were Amitermes amifer and Nasutitermes corniger. The functional group xylophages had the highest number of species (11) and the highest frequency. Species known as pests in eucalyptus had a frequency below the limit of dominance.
\end{abstract}

Index terms: Amitermes amifer, Eucalyptus grandis, Nasutitermes corniger, termites, xylophages.

\section{Introdução}

A crescente demanda por produtos de origem florestal tem levado à conversão de áreas nativas em florestas cultivadas, especialmente com o gênero Eucalyptus, o que favorece o surgimento de insetos praga, em razão da redução da complexidade biológica. Nessas plantações, de modo geral, destacam-se as formigas e os cupins como causadores de danos em larga escala (Holt \& Lepage, 2000).

Calderon \& Constantino (2007), bem como Varma \& Swaran (2007), no entanto, afirmam que o impacto negativo dos cupins em plantios de eucalipto, tem sido exagerado, e os benefícios que causam ao meio, subestimados e, muitas vezes, a simples presença desses insetos nas proximidades das plantas mortas é interpretada como a causa.

Apesar de algumas espécies de cupins causarem danos significativos, é importante salientar que, como em qualquer agroecossistema, uma das principais contribuições desses organismos ao ambiente é a ciclagem de nutrientes, essencial para manutenção de qualquer sistema ecológico (Black \& Okwakol, 1997).

Levantamentos sobre a termitofauna de área de silvicultura no Brasil são limitados e, muitas vezes, ignoram a existência de grupos funcionais diferentes (Junqueira et al., 2009). A falta de estudos sobre esses organismos, em especial quando são consideradas as florestas plantadas, é um entrave ao reconhecimento 
da importância dos cupins como insetos indispensáveis aos processos ecológicos nesses agroecossistemas.

O objetivo deste trabalho foi avaliar a frequência e a riqueza de espécies de cupins que ocorrem em plantações de eucalipto, na região do litoral norte da Bahia.

\section{Material e Métodos}

O estudo foi conduzido em três áreas de Eucalyptus grandis recém-colhido, da empresa Copener Florestal Ltda., nos municípios de Aramari, Santo André e Inhambupe, na região do litoral norte da Bahia. O clima da região é classificado como úmido a subúmido, com período chuvoso de fevereiro a agosto, pluviosidade anual média de $1.495,7 \mathrm{~mm}$ e temperatura média anual de $23,9^{\circ} \mathrm{C}$ (Superintendência de Estudos Econômicos e Sociais da Bahia, 2010).

As coletas foram realizadas após o corte das árvores de reflorestamento de eucalipto, com idade de sete anos, em dezembro de 2005 (Tabela 1). A forma do cultivo do reflorestamento foi em mosaico, com interação entre o reflorestamento e florestas nativas, reservas legais e reservas de preservação permanente.

Para a amostragem dos cupins, foi seguido o protocolo de avaliação rápida da biodiversidade de cupins, estabelecido por Jones \& Eggleton (2000), que consiste no estabelecimento de transectos em campo, com $100 \mathrm{~m}$ de comprimento por $2 \mathrm{~m}$ de largura, divididos em 20 parcelas contíguas de $5 \mathrm{~m}$ de comprimento cada uma e $2 \mathrm{~m}$ de largura. De cada parcela, foram coletadas aleatoriamente 12 amostras

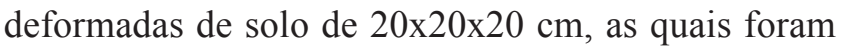
acondicionadas em recipientes e triadas em laboratório, no total de 240 amostras por área.

Os cupins foram identificados até a classificação de gênero, no Laboratório de Pragas Agrícolas e
Florestais, do Departamento de Ciências Florestais, da Universidade Federal de Sergipe, tendo-se utilizado a chave dicotômica proposta por Constantino (1999) e, posteriormente, até o nível de espécie, por meio de chaves, descrições, revisões de gêneros, ou por comparação com material depositado no Museu de Zoologia da Universidade de São Paulo. Os exemplares pertencentes às subfamílias Apicotermitinae e Nasutitermitinae, em alguns casos, foram separados em morfoespécies, pela dificuldade de identificação.

Os cupins foram classificados nos quatro grupos funcionais, de acordo com a literatura (Calderon \& Constantino, 2007; Reis \& Cancello, 2007; Carrijo et al., 2009): xilófagos, que se alimentam de madeira em diferentes estágios de decomposição; humívoros, que se alimentam de solo com matéria orgânica, ingerem grandes quantidades de húmus e geralmente vivem no perfil do solo; ceifadores ou comedores de folhas da serrapilheira, que cortam folhas ou se alimentam de pequenos fragmentos de madeira e outros itens da serrapilheira; e intermediários, cupins que são coletados predominantemente no solo, imediatamente sob troncos caídos ou colados a eles ou, ainda, dentro de troncos em avançado estágio de decomposição, onde já há solo misturado com madeira muito degradada.

Nas três áreas amostradas, a análise faunística foi realizada por cálculos de limite de dominância, a partir das equações: de dominância LD = $100(1 / \mathrm{S})$, proposta por Sakagami \& Laroca (1971), em que LD representa o limite de dominância, e $\mathrm{S}$ representa o número total de espécies; de frequência, em que $\mathrm{F}=100(\mathrm{n} / \mathrm{N})$; e de riqueza, pelo índice Jackknife de primeira ordem.

\section{Resultados e Discussão}

Foram coletadas 21 espécies de cupins, pertencentes a duas famílias e 16 gêneros, nas três áreas (Tabela 2).

Tabela 1. Áreas de estudo, suas coordenadas geográficas, altitude e descrição dos tipos de solo encontrados no litoral norte da Bahia.

\begin{tabular}{|c|c|c|c|c|}
\hline Área & Latitude & Longitude & Altitude (m) & Descrição do solo \\
\hline Aramari & $12^{\circ} 2^{\prime} 10^{\prime \prime S}$ & $38^{\circ} 32^{\prime} 58^{\prime \prime} \mathrm{W}$ & 0 & $\begin{array}{l}\text { Argissolo Amarelo distrocoeso arênico ou espessarênico, } \\
\text { abrúptico, Tb A fraco ou moderado, textura arenosa/ } \\
\text { média, relevo suave - ondulado e plano. }\end{array}$ \\
\hline Santo André & $12^{\circ} 1 ' 31 " \mathrm{~S}$ & $38^{\circ} 33^{\prime} 26^{\prime \prime} \mathrm{W}$ & 0 & $\begin{array}{l}\text { Argissolo Amarelo distrófico arênico ou espessarênico, } \\
\text { Tb A fraco ou moderado, textura arenosa/média, relevo } \\
\text { suave - ondulado e plano. }\end{array}$ \\
\hline Inhambupe & $11^{\circ} 51^{\prime} 5 " \mathrm{~S}$ & $38^{\circ} 28^{\prime} 31^{\prime \prime W}$ & 0 & $\begin{array}{l}\text { Latossolo Amarelo distrocoeso típico, Tb A fraco ou } \\
\text { moderado, textura média/média, relevo plano. }\end{array}$ \\
\hline
\end{tabular}

Pesq. agropec. bras., Brasília, v.45, n.12, p.1351-1356, dez. 2010 
O limite de dominância da área total foi de 4,35\%. Das 21 espécies observadas nas áreas de estudo no litoral norte da Bahia, 10 foram consideradas dominantes, todas pertencentes à família Termitidae, em que a espécie Amitermes amifer foi a de maior dominância da subfamília Termitinae, com $27 \%$ de frequência. Na subfamília Nasutitermitinae, as duas espécies que ultrapassaram o limite de dominância pertencem ao gênero Nasutitermes.

A riqueza observada de $\mathrm{S}=21$ foi considerada baixa, em comparação à encontrada em estudos realizados em mata nativa que adotaram o protocolo estabelecido por Jones \& Eggleton (2000). A comparação da riqueza de espécies da termitofauna, entre áreas de mata nativa $\mathrm{e}$ áreas de plantio de eucalipto, mostra que o número de espécies observadas em silvicultura, em geral, é menor em razão da simplificação das relações ecológicas e da pouca diversidade de alimento (Jones et al., 2003; Donovan et al., 2007), tendo sido observada tendência

Tabela 2. Lista de espécies observadas por família, frequência relativa (FR) e grupo funcional (GF) de cupins, amostrados em Aramari (A1), Santo André (A2) e Inhambupe (A3), no litoral norte da Bahia.

\begin{tabular}{|c|c|c|c|c|c|}
\hline \multirow[t]{2}{*}{ Espécie } & \multicolumn{3}{|c|}{ Áreas de estudo } & \multirow[t]{2}{*}{$\mathrm{FR}(\%)$} & \multirow[t]{2}{*}{$\mathrm{GF}^{(1)}$} \\
\hline & A1 & A2 & A3 & & \\
\hline \multicolumn{6}{|l|}{ Termitidae: Apicotermitinae } \\
\hline Anoplotermes sp.1 & $\mathrm{x}$ & $\mathrm{x}$ & - & 7,2 & $\mathrm{Hu}$ \\
\hline Rupititermes sp. & - & - & $\mathrm{x}$ & 0,5 & $\mathrm{Se}$ \\
\hline \multicolumn{6}{|l|}{ Termitidae: Nasutitermitinae } \\
\hline Diversitermes sp.1 & $\mathrm{x}$ & $\mathrm{x}$ & - & 0,9 & $\mathrm{Se}$ \\
\hline Nasutitermes sp.1 & $\mathrm{x}$ & $\mathrm{x}$ & - & 7,7 & $\mathrm{Xi}$ \\
\hline Nasutitermes sp.2 & $\mathrm{x}$ & $\mathrm{x}$ & - & 9,9 & $\mathrm{Xi}$ \\
\hline Nasutitermes sp.3 & - & - & $\mathrm{x}$ & 1,4 & $\mathrm{Xi}$ \\
\hline Nasutitermes corniger & $\mathrm{x}$ & $\mathrm{x}$ & $\mathrm{x}$ & 6,8 & $\mathrm{Xi}$ \\
\hline Constrictotermes cyphergaster & $\mathrm{x}$ & - & - & 4,1 & $\mathrm{Xi}$ \\
\hline Velocitermes sp.1 & $\mathrm{x}$ & $\mathrm{x}$ & $\mathrm{x}$ & 5,0 & $\mathrm{Se}$ \\
\hline Velocitermes heteropterus & $\mathrm{x}$ & - & $\mathrm{x}$ & 6,3 & Se \\
\hline Subulitermes microsoma & - & - & $\mathrm{x}$ & 0,5 & $\mathrm{Hu}$ \\
\hline \multicolumn{6}{|l|}{ Termitidae: Termitinae } \\
\hline Amitermes amifer & $\mathrm{x}$ & $\mathrm{x}$ & - & 27,0 & $\mathrm{Xi}$ \\
\hline Amitermes nordestinus & $\mathrm{x}$ & - & - & 0,9 & In \\
\hline Anoplotermes sp. & - & - & $\mathrm{x}$ & 0,5 & $\mathrm{Hu}$ \\
\hline Cylindrotermes sapiranga & $\mathrm{x}$ & - & - & 2,7 & $\mathrm{Xi}$ \\
\hline Dentispicotermes sp.1 & - & - & $\mathrm{x}$ & 1,4 & $\mathrm{Hu}$ \\
\hline Microcerotermes sp.1 & $\mathrm{x}$ & - & - & 5,0 & $\mathrm{Xi}$ \\
\hline Microcerotermes exiguus & $\mathrm{x}$ & - & - & 0,9 & $\mathrm{Xi}$ \\
\hline Termes sp. 1 & $\mathrm{x}$ & - & $\mathrm{x}$ & 5,4 & In \\
\hline \multicolumn{6}{|l|}{ Rhinotermitidae: Heterotermitinae } \\
\hline Heterotermes assu & $\mathrm{x}$ & - & - & 1,4 & $\mathrm{Xi}$ \\
\hline Heterotermes longiceps & $\mathrm{x}$ & - & - & 0,5 & $\mathrm{Xi}$ \\
\hline
\end{tabular}

${ }^{(1)} \mathrm{Xi}$, xilófago; In, intermediário; Se, serrapilheira; Hu, humívoro. à diminuição das espécies com o aumento do uso do solo (Junqueira et al., 2008).

A riqueza observada foi relativamente diversificada, em comparação aos trabalhos realizados em área de reflorestamento, com a mesma metodologia. Gutiérrez et al. (2004) utilizaram o protocolo proposto por Jones \& Eggleton (2000), para inspecionar somente os caules de espécies cultivadas (Eucalyptus tereticornis, Eucalyptus camaldulensis e Eucalyptus urophylla) na Colômbia, e encontraram apenas três espécies de cupins. Em estudo realizado por Varma \& Swaran (2007), em Kerala, Índia, com a mesma metodologia, foram encontradas 14 espécies de cupins em plantios de E. tereticornis.

A região do litoral norte da Bahia tem grande diversidade de ecossistemas e fitofisionomias, com áreas de floresta Ombrófila Densa e Estacional Semidecidual (Superintendência de Estudos Econômicos e Sociais da Bahia, 2010), cerrado e restinga, o que, de fato, justifica a existência de espécies animais endêmicas, características de ambientes preservados, a exemplo de Constrictotermes cyphergaster (Constantino, 1999). O agroecossistema de eucalipto aqui estudado foi cultivado em sistema de plantio em mosaico, com interação entre florestas nativas, reservas legais e reservas de preservação permanente com os cultivos.

Alguns gêneros de cupins, observados no presente estudo, já foram coletados em áreas de plantio de eucalipto em outros locais. Das sete áreas estudadas por Junqueira et al. (2009), no Município de Anhembi, SP, quatro eram de cultivo de Eucalyptus spp., das quais foram coletadas 13 espécies. Dos 12 gêneros encontrados pelos autores, cerca de três Dihoplotermes, Diversitermes e Nasutitermes - foram comuns aos do presente trabalho. No estudo realizado por Calderon \& Constantino (2007), em plantações de eucalipto em Buriti, MG, 10 gêneros foram comuns aos observados na região do litoral norte da Bahia.

Das duas espécies dominantes pertencentes à subfamília Apicotermitinae, a frequência de Anoplotermes sp.1 é atribuída à exaustiva exploração do solo, na metodologia de amostragem empregada durante as coletas realizadas. Espécies de Anoplotermes ocorrem em vários tipos de habitats, e a maioria delas vive em galerias difusas no solo, alimentandose de matéria orgânica em decomposição. Segundo 
Constantino (2002), A. pacificus é uma espécie causadora de danos em plantações de eucalipto.

As espécies da subfamília Nasutitermitinae são comumente registradas em áreas de cultivo de eucalipto. Das cinco espécies dominantes pertencentes a essa subfamília, três pertencem ao gênero Nasutitermes, que tem o maior número de espécies no mundo, com maior diversidade justamente na região neotropical (Constantino, 2009).

Nasutitermes corniger, observada como a terceira espécie em dominância, é relatada por Bandeira et al. (2003) como a mais frequente em áreas que já sofreram algum distúrbio, ainda não registrada em áreas de floresta primária. É uma das espécies de distribuição mais ampla, encontrada do sul do México até o norte da Argentina. NoBrasil, essecupimocorre em praticamente todo território nacional e é considerada a mais comum e importante praga do gênero Nasutitermes, inclusive em área urbana (Constantino, 2009).

Constrictotermes cyphergaster também foi a única espécie de ninho arborícola observada em plantios de eucalipto em Buritis, MG, por Calderon \& Constantino (2007), sem causar danos à plantação.

Na subfamília Termitinae, Amitermes teve a maior frequência (27\%), semelhantemente ao observado em estudo desenvolvido em duas áreas de Mata Atlântica, em diferentes estágios, em Ilhéus, BA, por Reis \& Cancello (2007). Segundo esses autores, isso se deve ao fato de as espécies desse gênero terem a capacidade de explorar a celulose em diferentes formas, bem como a madeira em diferentes estágios de decomposição. A. amifer é considerada praga secundária (Constantino, 2002, 2009) de diversas culturas como soja, amendoim e cana-de-açúcar.

Microcerotermes ocorre na Amazônia e no Cerrado, é encontrada em diversos habitats e possui algumas espécies consideradas pragas secundárias. Espécies desse gênero são as construtoras de ninho mais abundantes (Bandeira \& Vasconcelos, 2003) e, segundo Constantino (2002), a atividade de nidificação de Microcerotermes e de Nasutitermes pode causar danos às plantas, ao lesionar o tecido lenhoso. As espécies consideradas pragas, no Brasil, são: M. strunckii, praga secundária no cultivo de frutíferas; e $M$. arboreus, praga secundária em cultivo de maracujá. Contudo, a respeito da outra espécie desse gênero (não dominante), observada na região do litoral norte da Bahia $-M$. exiguus -, não foi encontrado nenhum registro de danos a plantações na literatura consultada.

Foram observadas espécies não dominantes da família Termitidae, consideradas pragas de acordo com a literatura, bem como espécies típicas de mata nativa. Amitermes nordestinus, conhecida como cupim miudinho, é considerada praga em lavouras de cana-de-açúcar e de abacaxi. Em estudo realizado no Estado da Paraíba, por Miranda et al. (2004), foi evidenciado seu potencial como praga em lavouras de cana-de-açúcar, tendo sido também relatados ataques a raízes de bromélias e cactos, em áreas de Caatinga e Cerrado do Nordeste do Brasil.

Cylindrotermes tem cinco espécies com distribuição neotropical e é típico de florestas, onde se alimenta de madeira morta sobre o solo. A espécie C. sapiranga, encontrada no presente estudo, tem distribuição no Norte e Nordeste do país (Rocha \& Cancello, 2007) e é considerada praga em cultivo de cana e eucalipto (Constantino, 2002).

Armitermes tem nove espécies, com ocorrência em quase todo o país, exceto na região Sul. Vive tanto em florestas como no Cerrado e em vegetações abertas. Alimenta-se de madeira em diferentes estágios de decomposição e de matéria orgânica semidecomposta em geral (Mathews, 1977; Constantino, 1999). A espécie encontrada, A. euamignathus, é citada por Calderon \& Constantino (2007) como causadoras de danos em plantações de eucalipto.

Cupins do gênero Subulitermes ocorrem em todo o Brasil, possuem hábito alimentar humívoro e vivem no solo ou em ninhos de outros cupins (Mathews, 1977; Constantino, 1999). S. microsoma também observada por Junqueira et al. (2004) numa floresta de E. pilularis, em Anhembi, SP, é considerada praga em plantações de eucalipto por Constantino (2002).

Cupins do gênero Heterotermes ocorrem em todas as regiões do Brasil, adaptam-se a diversos tipos de habitats e vivem, normalmente, em madeira ou em ninhos difusos no solo (Constantino, 1999). As espécies desse gênero, encontradas no presente trabalho, são consideradas pragas secundárias, como: $H$. assu, praga em área urbana e $H$. longiceps, em cana-de-açúcar e eucalipto (Constantino, 2002).

A partir dos valores obtidos pelo Jackniffe de primeira ordem, foram construídas curvas de riqueza, e observou-se que o esforço amostral foi suficiente para 
coletar a maior parte da fauna de cupins existente nas respectivas áreas (Figura 1).

Considerando-se as três áreas de estudo no litoral norte da Bahia, o grupo funcional com o maior número (11) de espécies foi o xilófago, seguido pelo humívoro (com cinco) (Tabela 3). O grupo de xilófago representou o maior número de espécies e de amostras.

Com o plantio de extensas áreas de eucalipto no litoral norte da Bahia, a fauna de Termitidae parece ter sido afetada de maneira diferente, pois o grupo xilófago foi favorecido por ter maior capacidade de resistência aos distúrbios ambientais e pela oferta de alimento existente no tipo de cultura estudado - o que corrobora

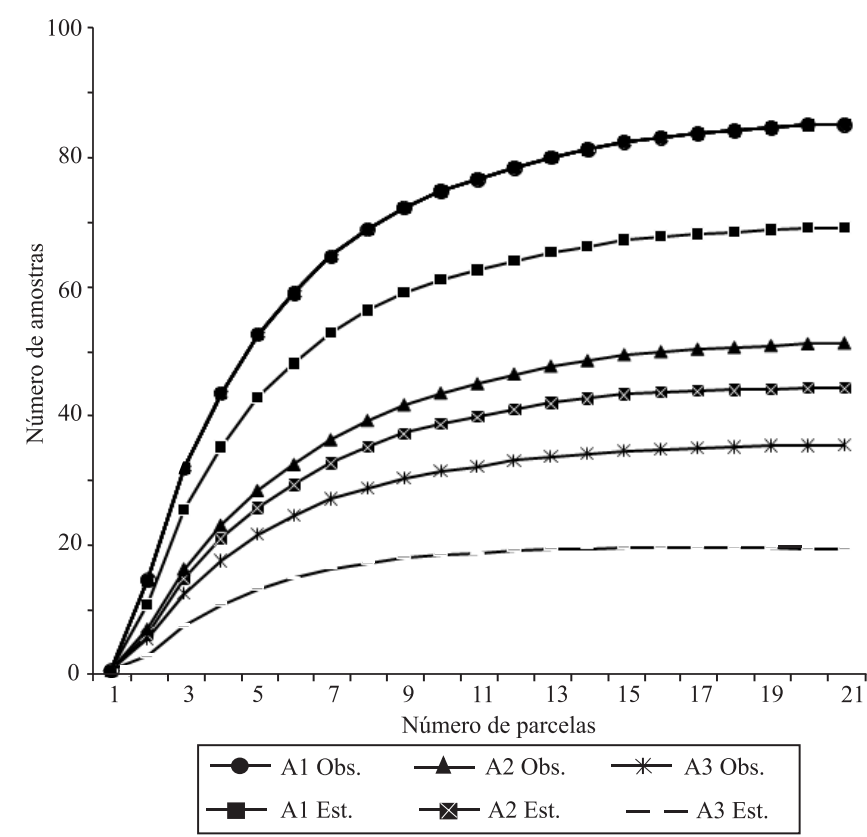

Figura 1. Curvas de riqueza observada (Obs.) e estimada (Est.), pelo índice Jackniffe de primeira ordem das espécies de cupins, em Aramari (A1), Santo André (A2) e Inhambupe (A3), no litoral norte da Bahia.

Tabela 3. Número de amostras de cupins, conforme o grupo funcional, coletado nas áreas de estudo do litoral norte da Bahia.

\begin{tabular}{lc}
\hline Grupo funcional & Total $(\%)$ \\
\hline Xilófago & 67,1 \\
Serrapilheira & 12,6 \\
Intermediário & 6,3 \\
Humívoro & 14,0 \\
\hline
\end{tabular}

os resultados de outros estudos (Eggleton et al., 1997; Jones \& Eggleton, 2000; Bandeira et al., 2003; Reis \& Cancello, 2007). Esses dados foram confirmados por Calderon \& Constantino (2007), que relataram que a conversão do cerrado em plantios de eucalipto levou à eliminação de muitos humívoros, enquanto a maioria dos xilófagos e ceifadores de serrapilheira permaneceu presente.

Nas áreas estudadas no litoral norte da Bahia, foram observados alguns gêneros que normalmente não são correlacionados a danos na silvicultura, e não são considerados pragas.

\section{Conclusões}

1. As espécies Amitermes amifer e Nasutitermes corniger têm a maior frequência nas áreas reflorestadas de eucalipto, e espécies conhecidas como pragas em eucalipto têm frequência abaixo do limite de dominância.

2. A riqueza de espécies de cupins, nas três áreas de reflorestamento de eucalipto no litoral norte da Bahia, é superior à encontrada em outras regiões de mesmo agroecossistema.

3. A riqueza de humívoros observada é considerada importante como indicação da manutenção de produtividade em plantações de eucalipto, o que evidencia que o papel ecológico desses insetos, nessas áreas, é relevante, apesar de pouco conhecido.

\section{Agradecimentos}

À Coordenação de Aperfeiçoamento de Pessoal de Nível Superior, por concessão de bolsa; à Copener Florestal, pelo suporte técnico; ao Museu de Zoologia da Universidade de São Paulo, pelo apoio na identificação do material.

\section{Referências}

BANDEIRA, A.G.; VASCONCELlOS, A.; SILVA, M.P.; CONSTANTINO, R. Effects of habitat disturbance on the termite fauna in a highland humid forest in the Caatinga domain, Brazil. Sociobiology, v.42, p.117-127, 2003.

BLACK, H.I.J.; OKWAKOL, M.J.N. Agricultural intensification, soil biodiversity and agroecosystem function in the tropics: the role of termites. Applied Soil Ecology, v.6, p.37-53, 1997.

CALDERON, R.A.; CONSTANTINO, R. A survey of the termite fauna (Isoptera) of an eucalypt plantation in central Brazil. Neotropical Entomology, v.36, p.391-395, 2007. 
CARRIJO, T.F.; BRANDÃO, D.; OLIVEIRA, D.E. de; COSTA, D.A.; SANTOS, T. Effects of pasture implantation on the termite (Isoptera) fauna in the central Brazilian savanna (Cerrado). Journal of Insect Conservation, v.13, p.575-581, 2009.

CONSTANTINO, R. Chave ilustrada para a identificação dos gêneros de cupins (Insecta: Isoptera) que ocorrem no Brasil. Papéis Avulsos de Zoologia, v.40, p.387-448, 1999.

CONSTANTINO, R. On-line termite database. Brasília: UnB, 2009. Disponível em: $<$ http://vsites.unb.br/ib/zoo/docente/constant/ catal/cat.htm>. Acesso em: 15 fev. 2010.

CONSTANTINO, R. The pest termites of South America: taxonomy, distribution and status. Journal of Applied Entomology, v.126, p.355-365, 2002.

DONOVAN, S.E.; GRIFFITHS, G.J.K.; HOMATHEVI, R.; WINDER, L. The spatial pattern of soil-dwelling termites in primary and logged forest in Sabah, Malaysia. Ecological Entomology, v.32, p.1-10, 2007.

EGGLETON, P.; HOMATHEVI, R.; JEEVA, D.; JONES, D.T.; DAVIES, R.G.; MARYATI, M. The species richness and composition of termites (Isoptera) in primary and regenerating lowland dipterocarp forest in Sabah, East Malaysia. Ecotropica, v.3, p.119-128, 1997.

GUTIÉRREZ, A.I.; URIBE, S.; QUIROZ, J.A. Termitas asociadas a plantaciones de Eucalyptus spp. en una reforestadora en Magdalena, Colombia. Manejo Integrado de Plagas e Agroecología, n.72, p.62-67, 2004.

HOLT, J.A.; LEPAGE, M. Termites and soil properties. In: ABE, T.; BIGNELL, D.E.; HIGASHI, M. (Ed.). Termites: evolution, sociality, symbiosis, ecology. Dordrecht: Kluwer Academic, 2000. p.389-407.

JONES, D.T.; EGGLETON, P. Sampling termite assemblages in tropical forests: testing a rapid biodiversity assessment protocol. Journal of Applied Ecology, v.37, p.119-203, 2000.

JONES, D.T.; SUSILO, F.X.; BIGNELL, D.E.; HARDIWINOTO, S.; GILLISON, A.N.; EGGLETON, P. Termite assemblage collapse along a land-use intensification gradient in lowland central Sumatra, Indonesia. Journal of Applied Ecology, v.40, p.380-391, 2003.

JUNQUEIRA, L.K.; DIEHL, E.; BERTI FILHO, E. Termite (Isoptera) diversity in Eucalyptus-growth areas and in forest fragments. Sociobiology, v.53, p.805-827, 2009.

JUNQUEIRA, L.K.; DIEHL, E.; BERTI FILHO, E. Termites in eucalyptus forest plantations and forest remnants: an ecological approach. Bioikos, v.22, p.3-14, 2008.

JUNQUEIRA, L.K.; DIEHL, E.; FLORENCIO, D.F.; BERTI FILHO, E. Seasonal species richness of termite in an Eucalyptus plantation in Anhembi, state of São Paulo, Brazil. Acta Biologica Leopoldensia, v.26, p.241-248, 2004.

MATHEWS, A.G.A. Studies on termites from the Mato Grosso state, Brazil. Rio de Janeiro: Academia Brasileira de Ciências, 1977. 267p.

MIRANDA, C.S.; VASCONCELlOS, A.; BANDEIRA, A.G. Termites in sugar cane in Northeast Brazil: ecological aspects and pest status. Neotropical Entomology, v.33, p.237-241, 2004.

REIS, Y.T.; CANCELLO, E.M. Riqueza de cupins (Insecta, Isoptera) em áreas de Mata Atlântica primária e secundária do sudeste da Bahia. Iheringia. Série Zoologia, v.97, p.229-234, 2007.

ROCHA, M.M. da; CANCELLO, E.M. Estudo taxonômico de Cylindrotermes Holmgren (Isoptera, Termitidae, Termitinae). Papéis Avulsos de Zoologia, v.47, p.137-152, 2007.

SAKAGAMI, S.F.; LAROCA, S. Relative abundance, phenology and flower visits of apid bees in Eastern Paraná, southern Brazil (Hymenoptera, Apoidea). Kontyü, v.39, p.217-230, 1971.

SUPERINTENDÊNCIA DE ESTUDOS ECONÔMICOS E SOCIAIS DA BAHIA. Estatística dos municípios baianos. Salvador: SEI, 2010. v.1, 414p.

VARMA, R.V.; SWARAN, P.R. Diversity of termites in a young eucalypt plantation in the tropical forests of Kerala, India. International Journal of Tropical Insect Science, v.27, p.95-101, 2007.

Recebido em 11 de julho de 2010 e aprovado em 26 de novembro de 2010 